Ann. Génét. Sél. anim., I974, 6 (3), 369-379.

\title{
VARIABILITÉ GÉNÉTIQUE ET CORRÉLATIONS PHÉNOTYPIQUES CARACTÉRISANT LA CONSOMMATION ALIMENTAIRE DE POULES PONDEUSES APRES CORRECTION POUR LE POIDS CORPOREL ET LA PONTE
}

\author{
A. BORDAS et P. MÉRAT \\ Laboratoire de Génétique factorielle, \\ Centre national de Recherches zootechniques, I. N.R. A., \\ 78350 Jouy en Josas
}

\section{RÉSUMÉ}

La consommation alimentaire individuelle de poules pondeuses a été enregistrée pendant 3 périodes successives de 28 jours dans des échantillons issus de 3 populations, de 1967 à 1973 . Nous considérons ici la variable (" consommation corrigée ") constituée par l'écart de cette consommation observée à la valeur obtenue à partir d'une équation de régression multiple sur le poids corporel, la variation de celui-ci et le poids d'œufs pondus pendant la période expérimentale. Pour ce critère, l'analyse de variance relative au facteur " famille de demi-sœurs " et l'estimation de la composante " entre familles " de la variance montrent l'existence d'une variabilité génétique non négligeable. D'autre part, des corrélations phénotypiques hautement significatives existent entre ce même critère et la consommation journalière d'eau, la taille des barbillons et de la crête, la longueur des tarses des pondeuses. L'interprétation suggérée tient compte de l'effet de ces dernières variables sur les déperditions énergétiques de l'oiseau. Du point de vue pratique, l'existence de variations génétiques intra-troupeau pour la consommation alimentaire " corrigée ", indice d'efficacité alimentaire, indique qu'une sélection directe sur ce critère serait concevable au moins dans certains troupeaux. Les corrélations observées suggèrent, de plus, la possibilité d'une amélioration indirecte relativement simple par sélection pour des appendices réduits et éventuellement par élimination d'animaux à consommation d'eau excessive.

\section{INTRODUCTION}

L'efficacité de la conversion de l'aliment par les poules pondeuses dépend pour une large part de leur production d'œufs et de leur poids corporel : par sélection augmentant la première et diminuant le second, des gains importants ont été réalisés 
sur le rendement des lignées et croisements spécialisés pour la ponte. Il peut cependant subsister, à poids corporel et à production d'œufs égaux, une variabilité " résiduelle " de la consommation d'aliment, correspondant à des différences dans l'ingestion et l'efficacité de l'utilisation des nutriments ingérés. Peu de travaux expérimentaux ont porté sur ce dernier point; en particulier peu d'observations existent sur la consommation individuelle d'aliment à l'intérieur d'une même population. Les résultats dans ce domaine ont été passés en revue par NoRDSKoG et al. (I972).

Nous avons voulu dans l'article qui suit rassembler nos résultats depuis I967, portant sur l'importance de la composante génétique et sur les corrélations phénotypiques associées à la consommation alimentaire de poules en ponte à l'intérieur de plusieurs populations, après correction pour l'influence du poids corporel, de la variation de poids et de la ponte. Les méthodes à cet effet ont été discutées par exemple, chez les volailles par ByERLy (I94I), ou chez d'autres espèces par SUTHERLAND (I965).

\section{MATÉRIEL ET MÉTHODES}

Une première série de lots expérimentaux, de 1967 à 1973, est issue du troupeau expérimental élevé à Jouy en Josas ; il s'agit d'une population de format moyen comportant une ségrégation à divers loci connus (notamment, en I972 et I973 le gène " cou nu ", ayant un effet appréciable sur la consommation alimentaire). Les autres lots proviennent d'une souche " ponte " de race $R$ hodeIsland originaire de la station expérimentale du Magneraud (souche " $M 99$ ").

En outre, dans la population de Jouy, nous considérons à part, en 1973 et I974, des poules possédant le gène de nanisme lié au sexe $d w$, issues des mêmes familles que les autres.

Dans tous les cas, l'élevage des jeunes est fait au sol jusqu'à l'âge de r6 semaines puis les animaux sont mis en cages individuelles. Le contrôle d'alimention commence sur des poules âgées de 8 mois à $\mathbf{I}$ an d'âge préalablement choisies (couples de sœurs différant pour un gène connu, issues des familles les plus nombreuses). Ce contrôle se poursuit pendant 3 périodes consécutives de 28 jours. Les poules reçoivent un aliment à 16 p. Ioo de protéines et 2520 kcal, d'énergie métabolisable par kg, sous forme de farine (dans la majorité des cas) ou de granulés (en I970 et I97I). La formule de l'aliment est identique d'une année sur l'autre.

Par animal et par période sont enregistrés la consommation alimentaire $O$, le poids corporel moyen $P$, sa variation $\Delta P$, différence entre les poids enregistrés à la fin et au début d'une même période, enfin le poids des œufs pondus $\mathrm{E}$.

Seuls sont considérés les animaux ayant toutes les mesures durant les trois périodes ; la mortalité en cours d'expérience était d'ailleurs, dans l'ensemble, relativement peu élevée. Les seuls résultats utilisés ici sont ceux portant sur la moyenne des trois périodes pour chaque variable.

Le tableau I récapitule les effectifs et caractéristiques principales des lots expérimentaux pour chaque population, y compris les valeurs moyennes des variables mentionnées ci-dessus.

La consommation " théorique " ou "espérée " T pour chaque individu est ensuite estimée en fonction de son poids moyen $\mathbf{P}$, de sa variation de poids $\Delta \mathbf{P}$ et du poids total d'œufs pondus $E$ par 28 jours suivant une équation de régression multiple :

$$
\mathrm{T}=a \mathrm{P}^{\alpha}+b \Delta \mathrm{P}+c \mathrm{E}
$$

du type défini par BYERLY (I94I). La méthode par approximations décrite par cet auteur, utilisée dans nos conditions, indique que l'on peut utiliser pour $\alpha$ une valeur voisine de 0,5 , et que d'ailleurs des variations assez larges autour de cette valeur modifient relativement peu les coefficients de l'équation obtenue. C'est cette valeur que nous avons retenue, par commodité, pour tous nos groupes expérimentaux. Dans chacun de ces groupes, la différence $\mathrm{R}=\mathrm{O}-\mathrm{T}$ entre consommation observée et théorique est appelée consommation "corrigée ". Parallèlement, une consommation " partiellement corrigée " $\mathrm{R}^{\prime}$ est calculée, différence entre la consommation observée et une consommation théorique $T^{\prime}$ calculée à partir d'une équation analogue à la précédente, mais n'incluant pas la variation de poids $\Delta P$. Alors que la consommation "corrigée " $R$ exprime un rendement, la variable $\mathrm{R}^{\prime}$ pourrait s'interpréter davantage comme un facteur d'ingestion alimentaire à poids et production donnés. 


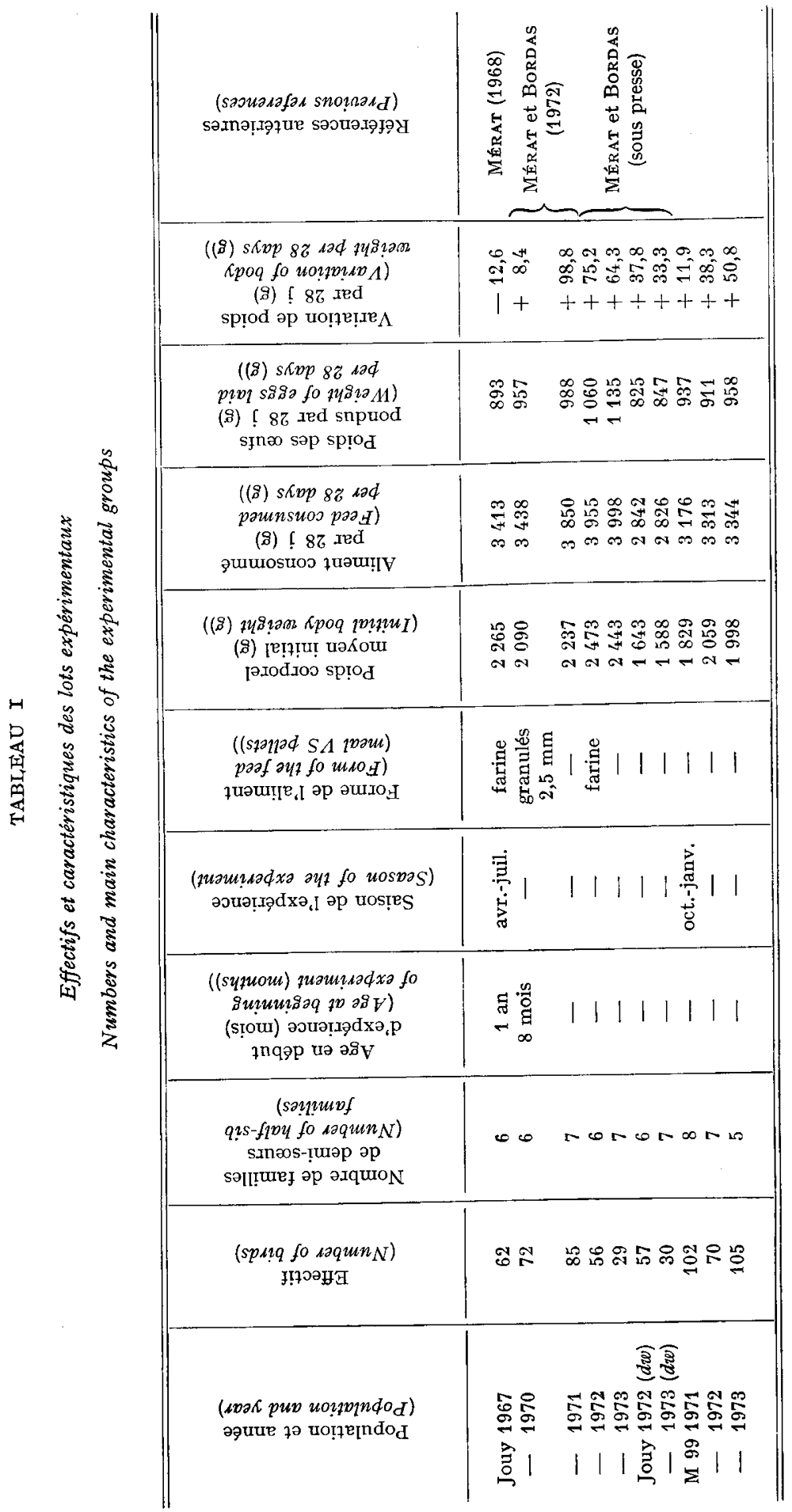


A partir de là, l'étude des résultats est faite séparément, sur chacune des trois variables distinctes $O, R$ et $R^{\prime}$. A titre indicatif, le tableau 2 fait apparaitre, pour chaque année et pour l'ensemble, les coefficients de l'équation de régression par population. Ces coefficients ne se sont pas révélés hétérogènes entre années et entre populations. Il en est de même du coefficient de corrélation multiple, dont la valeur estimée sur l'ensemble des données est o,79 (corresfondant à $63 \mathrm{p}$. Ioo de la variance de la consommation d'aliment expliqués par les variables $\sqrt{\mathrm{P}}, \Delta \mathrm{P}$ et $\mathrm{E})$.

TABLEAU 2

Équations de régression par années

Regression equations for each year

\begin{tabular}{|c|c|c|c|}
\hline \multirow{2}{*}{$\begin{array}{l}\text { Population et année } \\
\text { (Population and year) }\end{array}$} & \multicolumn{3}{|c|}{$\begin{array}{l}\text { Coefficient de } \\
\text { (Coefficient of) }\end{array}$} \\
\hline & $\mathrm{P}^{0,5}$ & $\Delta \mathrm{P}$ & $\mathbf{E}$ \\
\hline \multicolumn{4}{|l|}{ "Jouy "Dw: } \\
\hline 1967 & 58,3 & 1,63 & 0,76 \\
\hline 1970 & 65,0 & 3,04 & 0,70 \\
\hline 1971 & 60,6 & 2,11 & 0,97 \\
\hline 1972 & 84,0 & 2,68 & 1,14 \\
\hline 1973 & 94,8 & 3,70 & 0,23 \\
\hline Ensemble & 72,5 & 2,63 & 0,76 \\
\hline \multicolumn{4}{|l|}{ « Jouy " $d w:$} \\
\hline 1972 & 69,5 & 2,83 & 0,77 \\
\hline 1973 & 62,7 & 3,79 & 1,06 \\
\hline Ensemble & 66,1 & 3,31 & 0,91 \\
\hline \multicolumn{4}{|l|}{ " M 99 " : } \\
\hline 1971 & 53,6 & 3,24 & 1,07 \\
\hline 1972 & 71,5 & 3,49 & 1,08 \\
\hline 1973 & 78,5 & 1,28 & 0,74 \\
\hline Ensemble & 67,8 & 2,67 & 0,96 \\
\hline Total & 69,8 & 2,78 & 0,85 \\
\hline
\end{tabular}

Notre étude comporte d'abord une analyse de variance intra-années relative au facteur a famille de même père ", reproduisant en partie des résultats déjà publiés (Mł́RAT, I968; MÉRAT et BordAs, 1972, 1974). Lorsque des différences significatives se révèlent, associées aux génotypes à un locus connu étudié par ailleurs (cas, notamment, du gène " cou nu " en I972 et I973), 1'analyse est faite intra-génotype (aucune interaction entre génotype à un locus connu et famille ne s'est révélée significative). Cette analyse est présentée regroupée pour les diverses années de la population de Jouy (poules normales $D w$ d'une part, $d w$ de l'autre) et d'autre part de la souche $\mathbf{M} 99$. Nous avons, en outre, estimé une composante " entre familles de même père " de la variance et une composante " intra-familles", pour chaque variable de consommation, sans vouloir en tirer autre chose qu'un ordre de grandeur de l'héritabilité de cette variable, étant donné le nombre assez faible de mères pour chaque père et de descendants par mère dans nos échantillons. Certaines années (Jouy, I970-7I; I972-73; M 99 I97x-r972 et 72-73), des corrélations mères-filles ont pu être estimées sur une partie de ces mêmes échantillons.

Quant aux corrélations phénotypiques de la consommation individuelle observée, corrigée ou partiellement corrigée, elles ont été estimées avec une série d'autres variables représentatives du taux de ponte, des caractéristiques des œufs, de la croissance et du poids adulte. Nous y ajoutons la consommation journalière d'eau (moyenne mesurée sur I I jours en 1970, sur I6 jours les 
années suivantes à la fin de la $3^{\mathrm{e}}$ période du contrôle de consommation alimentaire) et des mensurations extérieures, longueur des tarses et longueur des barbillons à ro mois d'âge. Les caractères des œufs sont également observés vers ro mois. (Poids moyen sur 2 semaines, hauteur de l'albumen évaluée sur 2 œufs par poule après étalement sur une surface plane, épaisseur de coquille mesurée au comparateur, également sur $2 œ u f s$ par poule.) Concernant la longueur des barbillons, ses corrélations avec les variables de consommation ont été estimées intra-génotypes relatifs aux loci $\mathrm{P}$ et $\mathrm{Na}$, ces deux gènes modifiant sensiblement cette mensuration. La définition précise de chaque caractère est donnée au tableau 6.

\section{RÉSULTATS}

\section{I. - Variabilité génétique}

Le tableau 3 donne pour la consommation alimentaire observée ou corrigée, ainsi que pour la variation de poids, le rapport de la variance " entre familles de demisœurs " cumulée intra-années à la variance résiduelle intra-familles, pour les trois populations considérées et pour leur total : Dans tous les cas, et notamment pour la consommation " corrigée » ( $R$ ou $\left.R^{\prime}\right)$, les différences entre familles sont hautement significatives.

Le tableau 4 présente l'estimation du rapport de la composante " entre familles" de la variance à la variance totale. L'héritabilité estimée devant être comprise entre 2 fois et 4 fois cette valeur, on voit qu'elle atteint un ordre de grandeur très appréciable pour tous les caractères et dans les trois populations étudiées.

Le tableau 5 montre, pour les années où cela était possible, la corrélation entre valeur des mères et moyenne de leurs filles pour la consommation observée ou " corrigée ". Cette corrélation est positive, à une exception près, mais, du fait des effectifs réduits, 1'erreur d'échantillonnage dans chaque cas est élevée. En I970, 7 I et 72, la consommation alimentaire a été relevée également chez des coqs, frères ou demifrères des poules faisant l'objet du présent travail ; la variabilité chez les $\widehat{o} \sigma$ était élevée et la corrélation entre moyenne des frères et moyenne des sœurs s'est révélée faible. Les données sur les coqs ne sont pas présentées ici.

\section{2. - Corrélations phénotypiques}

La consommation alimentaire observée présente une corrélation importante avec le poids corporel de la poule $(r=+0,57)$ sur l'ensemble des données, sa production d'œufs $(r=+0,49)$ et sa variation de poids $(r=+0,42)$, comme on pouvait s'y attenđre et comme nous 1'avions déjà vérifié (cf. références citées au tableau I). De même, comme prévu, la consommation " partiellement corrigée » est en corrélation positive avec la variation du poids corporel au cours de l'expérience. Nous avons voulu mettre ici l'accent sur les corrélations de la consommation alimentaire " corrigée "indépendante du poids corporel, de sa variation et du poids d'œufs pondus - avec les caractéristiques de croissance et de ponte de l'animal. Le tableau 6 résume les résultats. Pour chaque couple de caractères, seule est présentée la corrélation estimée, sur une base intra-années, à partir de l'ensemble des années dans chaque population, après qu'un test ait confirmé l'absence d'hétérogénéité significative entre années 
A. BORDAS, P. MÉRAT

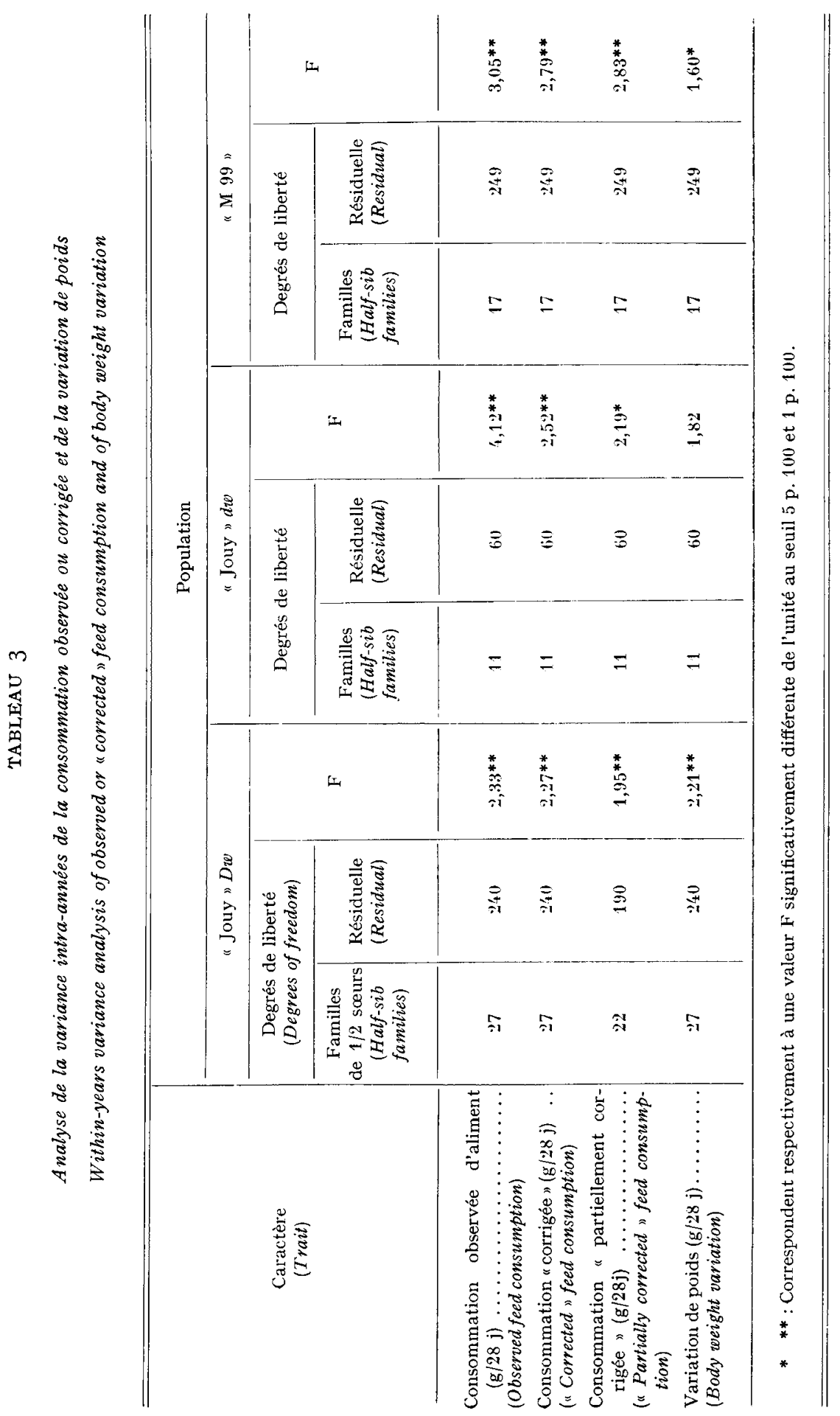


pour tous les coefficients de corrélation considérés. Nous avons fait également une estimation de ces coefficients sur l'ensemble des trois populations. Il n'y a pas non plus d'hétérogénéité significative entre ces populations, à l'exception de la corrélation avec la consommation d'eau (estimation sur le total mise entre parenthèses).

\section{TABLEAU 4}

Composante " entre familles " de la variance : vapport à la variance totale intra-années

« Between families "variance component : ratio to total within-year variance

\begin{tabular}{|c|c|c|c|}
\hline \multirow{2}{*}{$\begin{array}{l}\text { Caractère } \\
\text { (Trait) }\end{array}$} & \multicolumn{3}{|c|}{ Population (Population) } \\
\hline & "Jouy " Dw & "Jouy" dw & “M $99 "$ \\
\hline $\begin{array}{l}\text { Consommation observée } \ldots . . . \\
\text { (Observed feed consumption) }\end{array}$ & 0,123 & $0,33^{\prime} \mathbf{t}$ & 0,113 \\
\hline $\begin{array}{l}\text { Consommation corrigée ........ } \\
\text { ("Corrected" feed consumption) }\end{array}$ & 0,117 & 0,191 & 0,100 \\
\hline $\begin{array}{l}\text { Consommation partiellement } \\
\text { corrigée .............. } \\
\text { (" Partially corrected " feed } \\
\text { consumption) }\end{array}$ & 0,090 & 0,153 & 0,102 \\
\hline $\begin{array}{l}\text { Variation de poids } \ldots \ldots \ldots \ldots \ldots \\
\text { (Body weight variation) }\end{array}$ & 0,112 & 0,107 & 0,033 \\
\hline
\end{tabular}

TABLEAU 5

Corrélations mères-filles

Daughter-dam correlations

\begin{tabular}{|c|c|c|c|}
\hline \multirow[b]{2}{*}{ Caractère } & \multicolumn{3}{|c|}{ Population et année } \\
\hline & $\begin{array}{l}\text { Jouy } 72-73 \\
(n=18)\end{array}$ & $\begin{array}{c}\text { M } 99 \\
71-72 \\
(n=16)\end{array}$ & $\begin{array}{c}\text { M } 99 \\
72-73 \\
(n=30)\end{array}$ \\
\hline $\begin{array}{l}\text { Consommation observée } \ldots \ldots \ldots \\
\text { (Observed feed consumption) }\end{array}$ & $-0,02$ & $+0,71$ & $+0,11$ \\
\hline $\begin{array}{l}\text { Consommation corrigée ... } \\
\text { ("Corrected " consumption) }\end{array}$ & $+0,52$ & $+0,68$ & $+0,12$ \\
\hline $\begin{array}{l}\text { Consommation partiellement } \\
\text { corrigée .................. } \\
\text { (" Partially corrected" "consump- } \\
\text { tion) }\end{array}$ & $+0,16$ & $+0,53$ & $+0,05$ \\
\hline
\end{tabular}




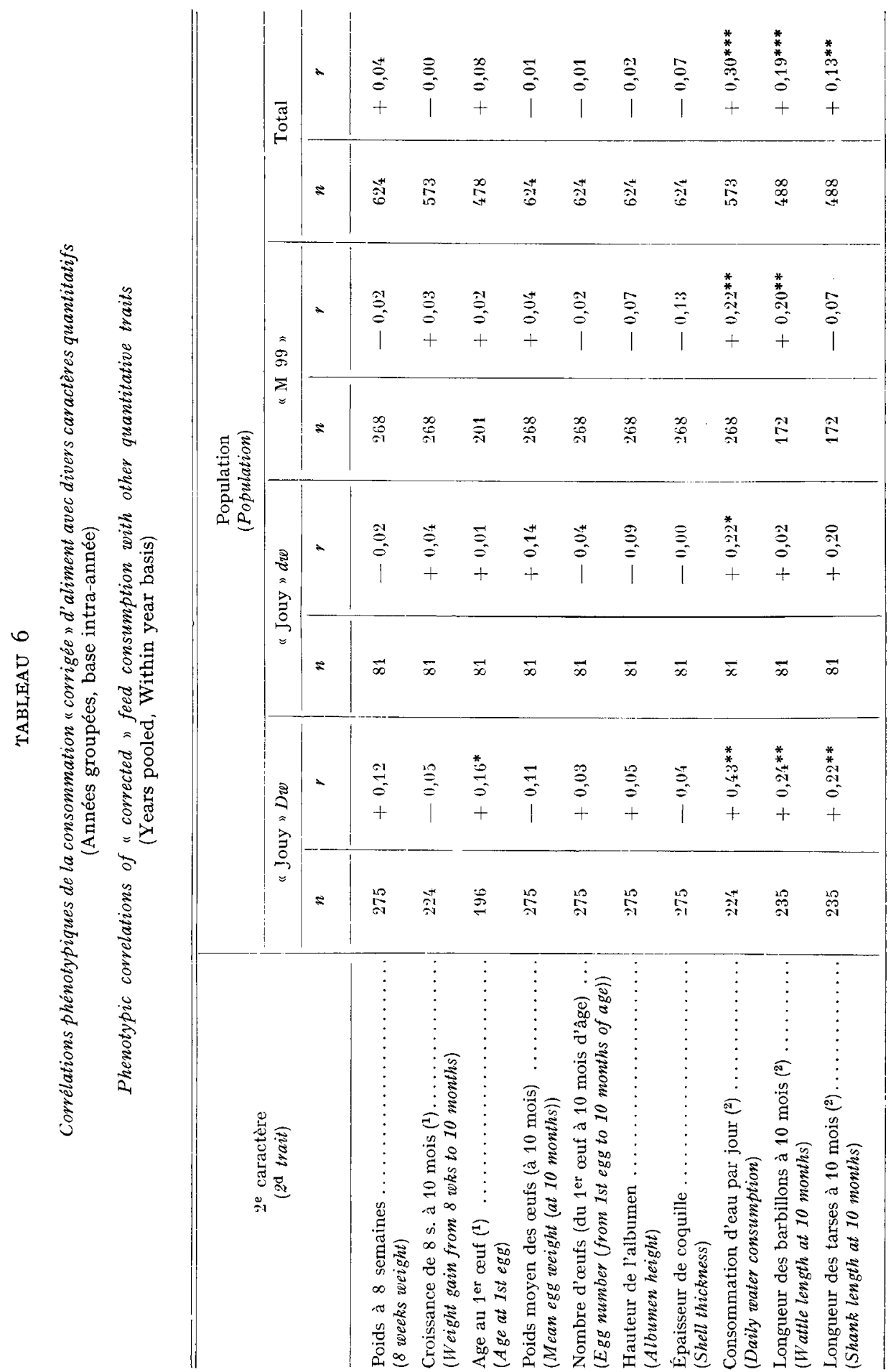




\section{DISCUSSION ET CONCLUSIONS}

Nos résultats font apparaître une variabilité génétique non négligeable de la fraction de la consommation alimentaire indépendante du poids et de la production de 1'animal, à en juger par les différences hautement significatives trouvées dans l'ensemble entre familles, et par la valeur de la composante "familles " de la variance. A l'inverse, NoRDskog et al. (I972) ne trouvaient pas de variations génétiques décelables pour ce caractère à l'intérieur de lignées de Leghorns. La différence pourrait s'expliquer par une hétérogénéité génétique plus grande de la population expérimentale utilisée à Jouy, et peut-être aussi de la souche $\mathrm{M} 99$, quoique cette dernière soit sélectionnée à la Station du Magneraud pour la production d'œufs depuis de nombreuses générations. On peut au moins conclure que, pour certaines populations, y compris des souches sélectionnées pour la ponte, une variabilité génétique existe pour la consommation alimentaire, non attribuable au format ni au taux de ponte. Dans de tels cas, une sélection directe ou indirecte sur ce caractère serait concevable.

Quant aux corrélations entre la consommation " corrigée » et d'autres caractères, trois, dans l'ensemble, méritent attention, celles avec la consommation d'eau, la taille des barbillons et la longueur des tarses des 우우 adultes.

Avec la consommation d'eau, la consommation " corrigée " $\mathrm{R}$ présente partout une corrélation positive relativement importante et hautement significative. Ceci suggère la possibilité d'améliorer indirectement le rendement alimentaire des pondeuses en sélectionnant pour une plus faible consommation d'eau, dans les cas où cette dernière serait d'une observation assez simple: ainsi pour un troupeau qui contiendrait le génotype récessif décrit par Dunson et Buss (I968) responsable d'une consommation d'eau excessive, les animaux correspondants étant décelables d'après la consistance de leurs déjections. Les coefficients de régression obtenus sur nos données laissent prévoir une diminution de consommation alimentaire de l'ordre de Ioo $\mathrm{g}$ par 28 jours pour Ioo $\mathrm{g}$ de moins d'eau ingérée par jour dans nos conditions.

On peut se demander dans quelle mesure la consommation d'eau est liée uniquement à la consommation globale d'aliment (par exemple si l'oiseau adapte la quantité d'eau bue à celle d'aliment ingéré). Or, le coefficient de corrélation partielle entre consommation " corrigée " $(\mathrm{R})$ et consommation d'eau, à consommation totale (O) d'aliment fixée, est respectivement de + o,I9 ( $\mathrm{P}<0, \mathrm{oI}),-0, \mathrm{I}_{3}$ (NS), 一 o,or (NS) pour les animaux " Jouy Dw", "Jouy dw " et " M 99 " (total des années) et de + 0,05 (NS) pour l'ensemble des données. Pour les poules naines et la souche M 99, la corrélation entre consommation corrigée d'aliment et consommation d'eau semble donc bien ne refléter qu'une corrélation globale entre ingestion d'aliment et d'eau, sans liaison de cette dernière avec un facteur de rendement alimentaire. Le cas semble différent pour la population "Jouy Dw». Or, la variabilité de la consommation d'eau y est nettement plus élevée que dans les deux autres, et nous y soupçonnons la présence d'un gène d'ingestion excessive d'eau identique ou analogue à celui đécouvert par Dunson et Buss. Peut-être un effet relativement limité de l'ingestion d'eau sur le rendement alimentaire a-t-il pu échapper à la détection dans les deux populations où le premier caractère était moins variable, à moins qu'un facteur de " polydipsie " 
n'ait un effet spécifique de ce point de vue. Pour expliquer un effet défavorable de l'ingestion accrue d'eau sur l'efficacité alimentaire, on pourrait invoquer l'augmentation des dépenses caloriques de l'animal causée par le réchauffement de l'eau ingérée jusqu'à la température corporelle ; il semble que cette hypothèse ne pourrait qu'en partie rendre compte de la corrélation de + 0,I9 observée dans la population " Jouy " et que d'autres causes d'ordre métabolique seraient à invoquer.

Les autres corrélations notables, celles de la consommation " corrigée " avec deux mensurations corporelles externes, la longueur des barbillons et celle des tarses, sont toutes deux hautement significatives dans l'ensemble, sans hétérogénéité décelable statistiquement entre les trois populations considérées. Pour fixer les idées, les coefficients de régression correspondants, sur l'ensemble des données, font prévoir un accroissement de III $\mathrm{g}$ de la consommation alimentaire par 28 jours, à poids et ponte égaux, pour I cm d'augmentation de la longueur des barbillons, et de ro5 $\mathrm{g}$ par $\mathrm{cm}$ en plus de longueur des tarses. Ces valeurs suggèrent la possibilité d'une amélioration indirecte du rendement alimentaire, dans des conditions comparables aux nôtres, en diminuant par sélection la taille des barbillons (et de la crête qui leur est liée) et celle des tarses. Ceci nous paraît explicable de façon simple par le biais des échanges énergétiques de l'oiseau ( $\left.{ }^{1}\right)$. En particulier dans le cas de la crête et des barbillons, on sait (STURKIE, I965) qu'une fraction relativement importante des déperditions caloriques de l'animal (évaluée à 15 p. Ioo en moyenne) a lieu par ces organes. Il est logique d'admettre que, toutes choses égales par ailleurs, les déperditions par cette voie sont en raison directe de la taille des appendices, et que cette dernière retentit donc sur le besoin énergétique, que l'on sait influer de façon prépondérante sur l'ingestion alimentaire. Pour les tarses, on peut suggérer une possibilité analogue, quoique l'importance des déperditions de chaleur par ces organes ne semble pas avoir été estimée ${ }^{2}$ ). On pourrait penser aussi à d'autres facteurs tels qu'une différence d'activité.

En fin de compte, nos résultats suggèrent qu'une sélection pour réduire les appendices non couverts par le plumage, sélection facile à réaliser, pourrait apporter indidirectement un gain limité mais cependant appréciable sur la fraction du rendement alimentaire des pondeuses ou des reproductrices indépendante de la taille corporelle et de la ponte, fraction qu'il serait long et coûteux d'améliorer par voie directe. Cette sélection ne semble pas comporter d'inconvénient par ailleurs, en l'absence de corrélations défavorables, dans nos conditions au moins, entre des appendices de taille réduite et les critères de production (MÉRAT, données non publiées). On peut espérer, peut-être, un gain analogue par élimination "à vue " des poules à consommation d'eau excessive. Par contre, les animaux sélectionnés dans ce sens pourraient être un peu moins adaptables en climats chauds.

Reçu pour publication en juillet 1974.

(1) On pourrait penser à un effet de la taille des barbillons sur le gaspillage d'aliment, mais ce dernier était très faible dans nos expériences.

$\left({ }^{2}\right)$ Wilson et Plaister (r95I) constatent cependant une corrélation négative entre la température corporelle et celle des tarses, de même que celle de la crête, et en concluent que ces organes jouent un rôle appréciable dans les déperditions caloriques. 


\title{
REMERCIEMENTS
}

Nous remercions M. Brbe (Station de Génétique quantitative et appliquée, C. N. R. Z., Jouy en Josas) et M. Guillaume (Station de Recherches avicoles, C. R. V. Z., Nouzilly) de leurs remarques et suggestions à la lecture de ce manuscrit.

\section{SUMMARY}

\author{
GENETIC VARIATION IN LAYING HENS AND PHENOTYPIC CORRELATIONS \\ OF FEED CONSUMPTION CORRECTED FOR BODY WEIGHT AND EGG PRODUCTION
}

Individual feed consumption of laying hens was measured during 3 successive 28 days periods in samples extracted from 3 populations, from 1967 to 1973 . We consider here the variable (" corrected consumption") defined by the deviation of observed consumption to the theoretical value obtained from a multiple regression equation on body weight, body weight variation and total weight of the eggs laid during the experimental period. For this criterion, the analysis of variance with the " half-sib families " factor (table 3) and the estimation of the "between families " component of variance (table 4) show the existence of an appreciable genetic variance. On the other hand, highly significant phenotypic correlations exist between this same criterion and daily water consumption, wattle and comb size, shank length of hens (table 6). A suggested explanation takes account of the effect of these latter variables on heat losses of the bird. From a practical point of view, the existence of genetic " within flock " variations for " corrected feed consumption ", taken as a criterion of feed efficiency, shows that a direct selection on this variable would be conceivable, at least in some flocks. The observed correlations suggest, moreover, the possibility of a relatively simple indirect improvement by selecting for appendages of reduced size and possibly by eliminating birds with excessive water consumption.

\section{RÉFÉRENCES BIBLIOGRAPHIQUES}

Byerly T. C., r94I. Feeds and other costs of producing market eggs. Univ. Maryland Agric. Exp. Sta., Bull. no Ar.

Dunson W. A., Buss E. G., 1968. Abnormal water balance in a mutant strain of chickens. Science 161, I67-I69.

Mérat P., I968. Consommation alimentaire de pondeuses $C c$ et $c c$. Ann. Zoot., 17, 337-339.

Mérat P., Bordas A., I972. Consommation alimentaire de pondeuses blanches (Ii) et colorées (ii). Ann. Génét. Sél. anim., 4., 375-384.

MÉrat P., Bordas A., r974. Consommation alimentaire d'animaux à plumage réduit (gène $\mathrm{Na}$ ) ou normal en la présence ou en l'absence du gène de nanisme dw. Ann. Génét. Sél. anim. (sous presse).

Nordskog A. W., French H. L. Jr, Arboleda C. R., Casey D. W. r972. Breeding for efficiency of egg production. World Poult. Sci. J., 29, х75-188.

Sturkie P. D., 1965. Avian physiology, $2^{\mathrm{e}}$ ed. Cornell University Press.

Sutherland T. M., I965. The correlation between feed efficiency and rate of gain, a ratio and its denominator. Biometrics, 21, 739-749.

Wilson W. O., Plaister T. H., r95I. Skin and feather temperatures of hens kept at constant environmental temperatures. Amer. J. Physiol., 166, 572-577. 